JPPT I Review

\title{
Efficacy of Bifidobacterium Species in Prevention of Necrotizing Enterocolitis in Very-Low Birth Weight Infants. A Systematic Review
}

Paige C. Hagen, BS, PharmD and Jessica W. Skelley, PharmD

Necrotizing enterocolitis (NEC) is one of the most common and serious gastrointestinal diseases in preterm infants. The aim of this systematic review examines the effects of probiotics on preventing NEC in verylow birth weight (VLBW) infants with a focus on the Bifidobacterium species and its strains. A systematic review of randomized trials and retrospective studies analyzing the use of probiotics to prevent NEC in VLBW infants was conducted using PubMed, Cochrane Central Registry of Controlled Trials, and Google Scholar (1996-2016). Trials reporting NEC involving preterm infants who were given Bifidobacterium alone in the first month of life were included in the systematic review. Nine studies were suitable for inclusion. Nine studies involving VLBW infants were analyzed for strain specific effects of Bifidobacterium for the prevention of NEC $\geq$ Stage II. $B$ breve showed some benefit in infants $<34$ weeks GA with relative risk (RR) of 0.43 (95\% confidence interval [Cl]: 0.21-0.87) $p=0.019$, but not in neonates $<28$ weeks. $B$ lactis greatly reduced the incidence of NEC with a RR 0.11 (95\% Cl: 0.03-0.47), $p=<0.001$. $B$ bifidum was not widely studied but resulted in no cases of NEC. Bifidobacterium proved to be statistically significant in reducing the incidence of NEC in preterm infants.

ABBREVIATIONS CFU, colony forming units; $\mathrm{Cl}$, confidence interval; NEC, necrotizing enterocolitis; NICU, neonatal intensive care unit; NNT, numbers needed to treat; RR, relative risk; VLBW, very-low birth weight

KEYWORDS necrotizing enterocolitis; preterm infant; probiotics; systemic review; very-low birth weight

J Pediatr Pharmacol Ther 2019;24(1):10-15

DOI: 10.5863/1551-6776-24.1.10

\section{Introduction}

Necrotizing enterocolitis (NEC) is one of the most common and devastating gastrointestinal emergencies in very-low birth weight (VLBW; $<1500 \mathrm{~g}$ ) infants in the neonatal intensive care unit (NICU). NEC is a multifactorial illness; it is a largely unpredictable disease in newborns and the etiology remains unclear despite advanced research.

The greatest risk factor for NEC is prematurity, putting preterm infants less than 37 weeks' gestation at the greatest risk. Risk factors associated with prematurity could account for this great risk, which include immunodeficiency, use of broad-spectrum antibiotics, delayed enteral feedings, and low availability of human milk. ${ }^{2}$ Other leading factors of NEC include hypoxia, feedings, abnormal colonization of the bowel, sepsis, and the release of inflammatory mediators that are stimulated by an ischemic-reperfusion injury in an immature gut. Tumor necrosis factor a and platelet-activating factors work together leading to an inflammatory cascade, contributing to mucosal damage. This inflammatory cascade is thought to be set off by events such as hypoxia in utero or sepsis. The inflammatory mediators signal neutrophil activation, increase the permeability of the vasculature, release reactive oxygen species, resulting in vasoconstriction with ischemic-reperfusion injury. Mucosal barriers continue to break down, leading to NEC of variable severities, which could lead to sepsis or death in some cases. ${ }^{3}$

Treatment is difficult and often inadequate, leading to a high rate of mortality and morbidity in neonates, making it an increasingly popular topic of research.' Ninety percent of NEC cases occur in preterm infants; however, it can still occur in near term or full term infants. Advances in medicine have resulted in the sustainability and support of lower gestational ages, but it has not decreased the incidence of NEC. Its incidence is indirectly related to gestational age with a wide variability among NICUs across the country.

Not only does NEC take a toll on mortality and morbidity, but it is associated with a high economic cost. It accounts for roughly $19 \%$ of neonatal expenditures and approximately $\$ 5$ billion per year for US hospitalizations. ${ }^{3}$ There is a great opportunity to make an impact on the total annual expenditure by implementing methods to prevent NEC in preterm infants. ${ }^{3}$

Recent research has focused on the immaturity of the infants' gut microbiota and manipulations of it. Feeding advancement and feeding content has been examined to help prevent NEC since enteral feedings are one of the only modifiable risk factors in NEC. The 
colonization patterns in a preterm infant's gut are distinctly different from those of a full-term infant. These differences are largely due to immaturity of intestinal epithelial glycoconjugate expression and the different environmental exposures experienced by preterm infants, leading to greater colonization of pathogenic bacteria. In addition to a longer exposure of pathogenic bacteria, preterm infants have a delayed acquisition of normal, nonpathogenic digestive flora, increasing their risk of developing NEC. ${ }^{4}$

For decades, probiotics have been studied for their potential health benefits of non-pathogenic microorganisms. They are live microorganisms, which when administered in adequate amounts, confer a health benefit on the host. The most common probiotic agents used today are Bifidobacterium, Lactobacillus, and Streptococcus. A number of randomized-controlled clinical trials have investigated the use of probiotics in preterm infants to reduce the incidence of NEC, focusing on normalizing intestinal flora. The rationale behind probiotic use relates to its identified benefits such as maintaining mucosal barrier integrity, activating intestinal immune defenses, regulating appropriate bacterial colonization, and controlling intestinal inflammation. ${ }^{4}$

The use of probiotics for the prevention of NEC in VLBW infants has been extensively studied in numerous randomized-controlled clinical trials. Their results have been summarized in several meta-analyses and systemic reviews, which show that probiotics reduce the rate of NEC and mortality among preterm infants. ${ }^{5,6}$ The authors strongly encourage the implementation of probiotic use in this population"; however, the American Academy of Pediatrics stressed the need for more research to determine the amount and specificity of probiotics that should be used. The beneficial effects of probiotics appear to be strain specific ${ }^{8}$ and combining all of the results from clinical trials can produce inaccurate and misleading recommendations because different strains were used in different trials. Aceti et $\mathrm{al}^{9}$ recently published a systematic review and meta-analysis that broke down the results based on different probiotic strains. It showed that the effects of Lactobacillus or Saccharomyces were not significant in reducing the incidence of NEC. However, Bifidobacterium showed a significant reduction in the incidence of NEC. Bifidobacterium is the predominant fecal bacteria in breastfed infants and the colonization by Bifidobacterium is delayed in preterm infants. ${ }^{10,11}$ Aceti et al ${ }^{9}$ used combination products with Bifidobacterium that contained Lactobacillus and/or Streptococcus, which could be a limitation of that meta-analysis, skewing the results. In comparison, this systematic review includes 2 studies that were not included in Aceti et $\mathrm{al}^{9}$ and is focused on the species of Bifidobacterium alone.

Current studies that have reported on all probiotics collectively lack species specificity and gut colonization data. $^{12-15}$ It is necessary to assess individual species and strains to determine which probiotic will confer the best health benefits in preterm infants. ${ }^{16-18}$ The aim of this systematic review is thus to evaluate in detail the effects of probiotics for the prevention of NEC in VLBW preterm infants, with a focus on Bifidobacterium and its strains.

\section{Materials and Methods}

Study Selection. A systematic review of published studies reporting the use of probiotics, specifically Bifidobacterium, for the prevention of NEC in preterm infants was performed. Criteria for inclusion in the systematic review were the following: randomized and quasi-randomized controlled trials or retrospective cohort studies involving preterm infants (gestational age $<37$ weeks) or VLBW infants (<1500 g) and reported on NEC (any stage according to Bell staging criteria ${ }^{19}$ ); enteral administration of probiotics from the Bifidobacterium species, compared with placebo or no treatment. The search was specifically focused on NEC so data on different outcomes, such as sepsis or mortality that were included in the studies, were not evaluated by the systematic review. Studies that used a combination product with Bifidobacterium were excluded.

A search was conducted in PubMed (http://www. ncbi.nlm.nih.gov/pubmed/) for studies published before October 14, 2016, using Medical Subject Heading terms "neonate," "infant, newborn," "very low birth weight," "probiotic," "Bifidobacterium," and "necrotizing enterocolitis." The Cochrane Library was searched using the same methods as for the PubMed database. The review was limited to studies written in English and that involved human subjects. Additionally, a manual search for different relevant studies and published reviews took place using Google Scholar.

Data Extraction. Studies retrieved from the searches were assessed for eligibility, data pertaining to the patients, intervention, control groups, type of feedings, outcomes, and methodology. The clinical outcome of interest was NEC $\geq$ Stage II according to the Bell staging criteria.

Statistical Analysis. For each study trial, relative risk (RR) with a $95 \%$ confidence interval (95\% Cl), and numbers needed to treat (NNT) for NEC were analyzed (Table). It was considered statistically significant if $\mathrm{p}<$ 0.05 .

\section{Results}

Literature Search. A total of 24 studies were selected for further review. Nine of these studies were selected to be included in the systematic review due to their inclusion criteria, probiotic choice, and clinical outcome of NEC. The preterm infants were divided into 2 groups: the probiotic group and the control group. A description of included studies is provided in the Table.

All of the studies reported NEC data suitable for a 


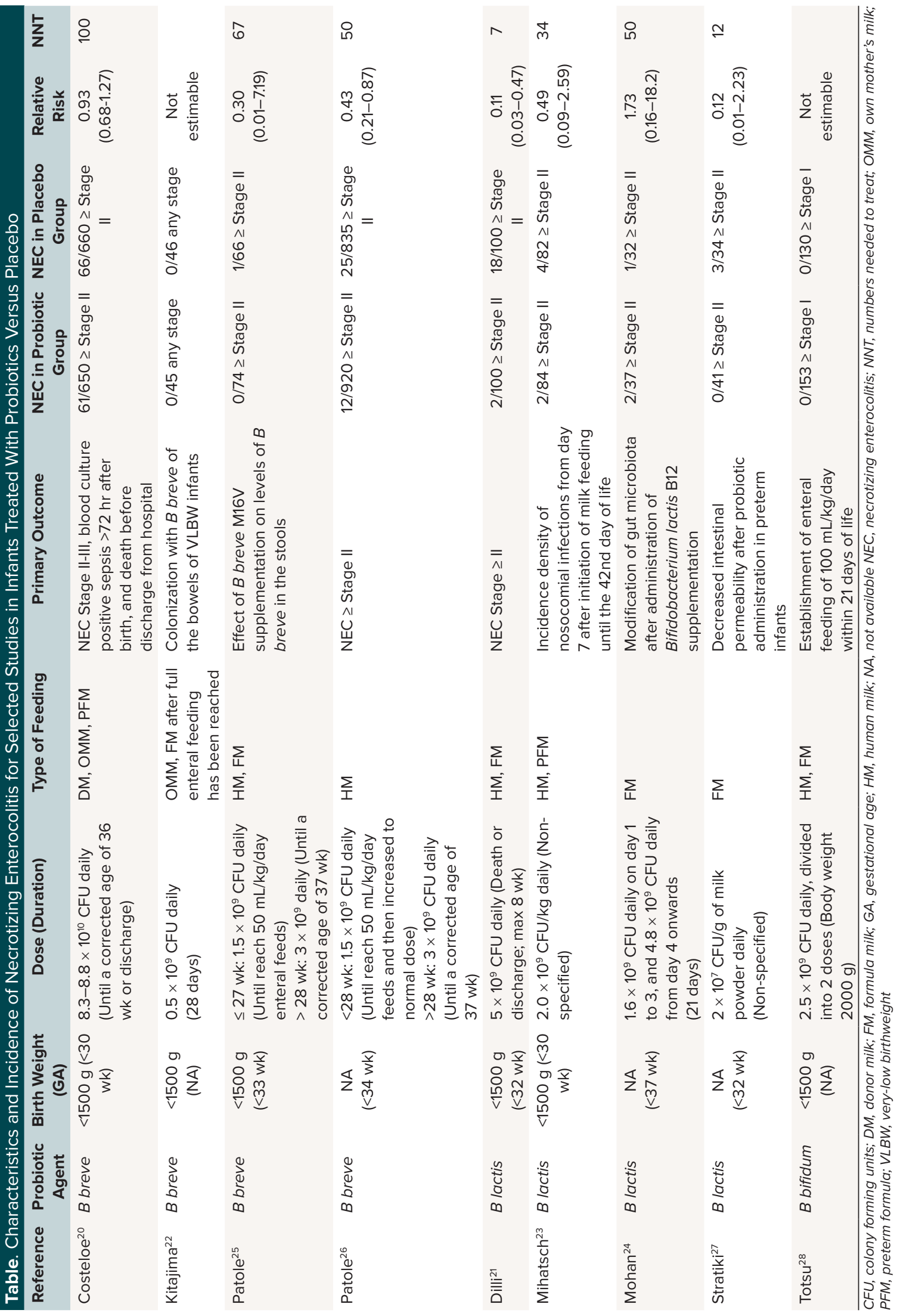


systematic review, except one, ${ }^{24}$ for which data were gathered from a previous Cochrane review. ${ }^{29}$ Data for each study's NEC rate in the probiotic versus the placebo group are shown in the Table. NEC $\geq$ Stage II data were used in this systematic review.

Probiotic Strain. Bifidobacterium breve was used in 4 studies. ${ }^{20,22,25,26}$ The effects of this probiotic strain in reducing NEC were not significant. Specifically, Costeloe et $\mathrm{al}^{20}$ and Patole et $\mathrm{al}^{25}$ showed no benefit. Costeloe et $\mathrm{al}^{20}$ used a dose of 8.3 to $8.8 \times 10^{10}$ colony forming units (CFU) daily, which resulted in RR 0.93 (95\% Cl: $0.68-1.27)$, $p$ value not specified. Patole et $\mathrm{al}^{25}$ had 2 different dosing protocols based on gestational age. For infants $>27$ weeks, the dose was $3.0 \times 10^{9} \mathrm{CFU}$ daily and $1.5 \times 10^{9} \mathrm{CFU}$ daily for newborns $\leq 27$ weeks until they reached $50 \mathrm{~mL} / \mathrm{kg} /$ day of enteral feeds. This resulted in a $\mathrm{RR}$ of 0.30 (95\% $\mathrm{Cl}$ : 0.01-7.19), $\mathrm{p}=<0.497 .{ }^{25}$ Kitajima et $\mathrm{al}^{22}$ showed positive colonization of the immature bowel with $B$ breve $0.5 \times 10^{9} \mathrm{CFU}$ daily, resulting in decreased incidence of abnormal abdominal signs, such as less gas accumulation and incidences of vomiting. Patole et $\mathrm{al}^{26}$ showed benefit in the reduction of NEC in infants $>28$ weeks supplemented with $3.0 \times 10^{9} \mathrm{CFU}$ daily. This resulted in a RR $0.43(95 \% \mathrm{Cl}$ : $0.21-0.87) p=0.019$. However, it did not show statistical significance in neonates $<28$ weeks who received 1.5 $\times 10^{9} \mathrm{CFU}$ daily until they reached feeds of $50 \mathrm{~mL} / \mathrm{kg} /$ day and then were increased to the normal dose. After pooling the studies, only 73 out of 1689 neonates who received $B$ breve developed NEC, which is about $4 \%$.

Bifidobacterium lactis was used in 4 studies. ${ }^{21,23,24,27}$ Bifidobacterium lactis resulted in a significant reduction in the risk for NEC. Dilli et $\mathrm{al}^{21}$ supplemented with $5.0 \times$ $10^{9} \mathrm{CFU}$ daily resulting in a RR 0.11 (95\% Cl: 0.03-0.47), $\mathrm{p}=<0.001$, with NEC incidence of $2 \%$ vs $18 \%$ for treatment versus placebo. Stratiki et $\mathrm{al}^{27}$ used $2.0 \times 10^{7}$ CFU/g of milk powder daily, which showed a RR 0.12 (95 \% Cl: 0.01-2.23), p value not specified. There were no occurrences of NEC in the probiotic group and an $8.8 \%$ occurrence in the placebo group. Mohan et $\mathrm{al}^{24}$ reported increased colonization of $B$ lactis in the infants' abdomen and saw reduced cell counts of enterobacteria and clostridia using a titrated daily dose of $1.6 \times$ $10^{9} \mathrm{CFU}$ on day 1 to 3 , and $4.8 \times 10^{9} \mathrm{CFU}$ daily from day 4 onwards with a RR $1.73(95 \% \mathrm{Cl}$ : 0.16-18.2), p value not specified. Mohan et $\mathrm{al}^{24}$ did not show benefit in the probiotic group versus the placebo group with NEC incidences of $5 \%$ vs 3\%. Additionally, Mihatsch et $\mathrm{al}^{23}$ showed no statistical benefit of $B$ lactis on NEC after supplementation with $2.0 \times 10^{9} \mathrm{CFU} / \mathrm{kg}$ daily resulting in a RR 0.49 (95\% Cl: 0.09-2.59), p value not specified. The reported incidences of NEC in the probiotic versus placebo group were $2 \%$ vs $4.9 \%$. Overall, 262 neonates received $B$ lactis, but only 6 of those infants developed NEC.

The only study to use the $B$ bifidum strain was conducted by Totsu et al. ${ }^{28}$ The authors showed earlier colonization of $B$ bifidum in neonates, with no cases of NEC in either the probiotic or placebo groups after supplementing with $2.5 \times 10^{9} \mathrm{CFU}$ daily, divided into 2 doses.

\section{Discussion}

NEC continues to be a challenging disease for VLBW neonates associated with high rates of mortality, morbidity, and cost. Despite advancing research, the etiology remains unclear. Due to insufficient treatment, it is one of the most common gastrointestinal emergencies in the preterm neonatal population. . Although there have been quality systemic reviews and recent publications that support the use of probiotics for the prevention of NEC in preterm infants, the use of probiotics has not been implemented into practice. This systematic review was conducted to provide more data supporting the use of probiotics, specifically Bifidobacterium as an intervention to prevent NEC. There is a need to determine which probiotic strain and dose provides the greatest benefit to neonates at high risk of developing NEC. High-quality studies were chosen based on their blinding to interventions and use of a single probiotic agent.

In regard to dosing of probiotics, it is important to achieve an optimal amount in order for the bacteria to survive and colonize in the gut in adequate amounts to confer a health benefit. Based on evidence, the suggested dose should minimally contain $10^{6}$ to $10^{7} \mathrm{CFUs}$ per gram of the product. ${ }^{30,31}$ This systematic review looked at 3 different strains of Bifidobacterium: breve, lactis, and bifidum. When comparing the results of the different Bifidobacterium strains, $B$ lactis showed the greatest statistically significant decrease in the incidences of NEC in the trial by Dilli et al. ${ }^{21}$ Overall, there were conflicting conclusions from the studies on their benefit in reducing NEC when given a probiotic versus placebo alone. There have been no observable risks associated with the probiotics; however, little is known about the long-term effects of altering the flora in the gut of preterm infants.

The decision to investigate a single probiotic species could be viewed as a limitation to the study; however, this was a deliberate choice seeing that further species and strain specific information is needed to determine its ability to prevent NEC. All trials reviewed met or exceeded the minimum daily dose; however, there was no standardized dose, limiting the conclusions that can be drawn from these trials. An additional limitation is the choice to focus only on NEC as the primary outcome. In the neonatal population, NEC often goes hand in hand with sepsis and increases infant mortality and morbidity. Past meta-analyses reported on the outcomes of NEC in addition to sepsis and all-cause mortality and morbidity; however, no benefit with probiotic supplementation was shown on decreasing the occurrence of sepsis. Furthermore, the inconsistencies in types of neonatal 
feedings between the studies can be seen as a limitation because the types of feedings are influential on the development of gut microbiota in preterm infants. It is well known that infants fed with either maternal or donor breast milk are associated with lower incidences of NEC when compared to formula-fed infants. The differences in feeding regimens between studies could have been a confounding factor, skewing the results. ${ }^{9}$

Although this systematic review demonstrates some benefit of Bifidobacterium on the prevention of NEC, unanswered questions remain. Future studies need to be aimed at determining any long-term effects of probiotic supplementation in VLBW infants and at solidifying the most effective probiotic species and strain, including its dose and duration of treatment to identify the best single or combination product for the prevention of NEC.

\section{ARTICLE INFORMATION}

Affiliations McWhorter School of Pharmacy (PCH, JWS), Samford University, Birmingham, AL

Correspondence Jessica W. Skelley, PharmD; jmwhalen@samford.edu

Disclosure The authors declare no conflicts or financial interest in any product or service mentioned in the manuscript, including grants, equipment, medications, employment, gifts, and honoraria.

\section{Accepted July 15, 2018}

Copyright Published by the Pediatric Pharmacy Advocacy Group. All rights reserved.

For permissions, email: matthew.helms@ppag.org

\section{REFERENCES}

1. Lin PW, Stoll BJ. Necrotising enterocolitis. Lancet. 2006;368(9543):1271-1283.

2. Sanders ME, Guarner F, Guerrant R, et al. An update on the use and investigation of probiotics in health and disease. Gut. 2013;62(5):787-796.

3. Gephart SM, McGrath JM, Effken JA, Halpern MD. Necrotizing enterocolitis risk: state of the science. $A d v$ Neonatal Care. 2012;12(2):77-89.

4. Martin CR, Walker WA. Probiotics: role in pathophysiology and prevention in necrotizing enterocolitis. Semin Perinatol. 2008;32(2):127-137.

5. Deshpande G, Rao S, Patole S, Bulsara M. Updated meta-analysis of probiotics for preventing necrotizing enterocolitis in preterm neonates. Pediatrics. 2010;125(5):921-930.

6. Alfaleh K, Anabrees J, Bassler D. Probiotics for prevention of necrotizing enterocolitis in preterm infants. Cochrane Database Syst Rev. 2014;4:CD005496.

7. Thomas DW, Greer FR. Probiotics and prebiotics in pediatrics. Pediatrics. 2010;126(6):1217-1231.
8. Szajewska H. Probiotics and prebiotics in preterm infants: where are we? Where are we going? Early Hum Dev. 2010;86(suppl 1):81-86.

9. Aceti A, Gori D, Barone G, et al. Probiotics for prevention of necrotizing enterocolitis in preterm infants: systematic review and meta-analysis. Italian J Pediatr. 2015;41:89.

10. Hammerman C, Bin-Nun A, Kaplan M. Germ warfare: probiotics in defense of the premature gut. Clin Perinatol. 2004;31(3):489-500.

11. Mohan R, Koebnick C, Schildt J, et. al. Effects of bifidobacterium lactis bb12 supplementation on intestinal microbiota of preterm infants: a double-blind, placebo controlled, randomized study. J Clin Microbiol. 2006;44(11):4025-4031.

12. Roy A, Chaudhuri J, Sarkar D, et al. Role of enteric supplementation of probiotics on late-onset sepsis by candida species in preterm low birth weight neonates: a randomized, double blind, placebo-controlled trial. $N$ Am J Med Sci. 2014;6(1):50-57.

13. Fernández-Carrocera LA, Solis-Herrera A, CabanillasAyón $\mathrm{M}$, et al. Double-blind, randomised clinical assay to evaluate the efficacy of probiotics in preterm newborns weighing less than $1500 \mathrm{~g}$ in the prevention of necrotising enterocolitis. Arch Dis Child Fetal Neonatal Ed. 2013;98(1):F5-F9.

14. Braga TD, da Silva GA, de Lira PI, de Carvalho LM. Efficacy of bifidobacterium breve and lactobacillus casei oral supplementation on necrotizing enterocolitis in very-lowbirth-weight preterm infants: a double-blind, randomized, controlled trial. Am J Clin Nutr. 2011;93(1):81-86.

15. Rougé $\mathrm{C}$, Piloquet $\mathrm{H}$, Butel $\mathrm{MJ}$, et al. Oral supplementation with probiotics in very- low-birth-weight preterm infants: a randomized, double-blind, placebo-controlled trial. Am J Clin Nutr. 2009;89(6):1828-1835.

16. Hill C, Guarner F, Reid G, et al. Expert consensus document: the international scientific association for probiotics and prebiotics consensus statement on the scope and appropriate use of the term probiotic. Nat Rev Gastroenterol Hepatol. 2014;11(8):506-514.

17. Food and Agriculture Organization of the United Nations, World Health Organization. Probiotics in food. Health and nutritional properties and guidelines for evaluation. FAO, Rome, Iral. Paper 85. City, ST or Country: Publisher; 2006. Report xx. http://www.fao.org/3/a-a0512e.pdf . Accessed January 29,2019.

18. Al-Hosni M, Duenas M, Hawk M, et al. Probiotics-supplemented feeding in extremely low-birth-weight infants. $J$ Perinatol. 2012;32(4):253-259.

19. Bell MJ, Ternberg JL, Feigin RD, et al. Neonatal necrotizing enterocolitis. Therapeutic decisions based upon clinical staging. Ann Surg. 1978;187(1):1-7.

20. Costeloe K, Hardy P, Juszczak E, et al. Probiotics in preterm infants study: collaborative g. bifidobacterium breve BBG-001 in very preterm infants: a randomised controlled phase 3 trial. Lancet. 2016;387(10019):649-660.

21. Dilli D, Aydin B, Fettah N, et al. The propre-save study: effects of probiotics and prebiotics alone or combined on necrotizing enterocolitis in very low birth weight infants. J Pediatr. 2015;166(3):545-551. 
22. Kitajima H, Sumida Y, Tanaka R, et al. Early administration of bifidobacterium breve to preterm infants: randomised controlled trial. Arch Dis Child Fetal Neonatal Ed. 1997;76(2):F101-F107.

23. Mihatsch WA, Vossbeck S, Eikmanns B, et al. Effect of bifidobacterium lactis on the incidence of nosocomial infections in very-low-birth-weight infants: a randomized controlled trial. Neonatology. 2010;98(2):156-163.

24. Mohan R, Koebnick C, Schildt J, et al. Effects of bifidobacterium lactis $\mathrm{Bb} 12$ supplementation on intestinal microbiota of preterm infants: a double-blind, placebo-controlled, randomized study. J Clin Microbiol. 2006;44(11):4025-4031.

25. Patole $S$, Keil AD, Chang A, et al. Effect of bifidobacterium breve $\mathrm{m}-16 \mathrm{v}$ supplementation on fecal bifidobacteria in preterm neonates - a randomised double blind placebo controlled trial. PLoS ONE. 2014;9(3):e89511.

26. Patole SK, Rao SC, Keil AD, et al. Benefits of bifidobacterium breve $\mathrm{m}-16 \mathrm{v}$ supplementation in preterm neonates - a retrospective cohort study. PLOS ONE. 2016;11(3):e0150775.
27. Stratiki Z, Costalos C, Sevastiadou S, et al. The effect of a bifidobacter supplemented bovine milk on intestinal permeability of preterm infants. Early Hum Dev. 2007;83(9):575-579.

28. Totsu S, Yamasaki C, Terahara M, et al. Bifidobacterium and enteral feeding in pre-term infants: cluster-randomized trial. Pediatr Int. 2014;56(5):714-719.

29. Alfaleh K, Anabrees J, Bassler D. Probiotics for prevention of necrotizing enterocolitis in preterm infants. Cochrane Database Syst Rev. 2011;3:CD005496.

30. Kosin B, Rakshit S. Microbial and processing criteria for production of probiotics: a review. Food Technol Biotechnol. 2006;44:371-379.

31. Deshpande G, Rao S, Keil A, Patole S. Evidence-based guidelines for use of probiotics in preterm neonates. BMC Med. 2011;9:92. 